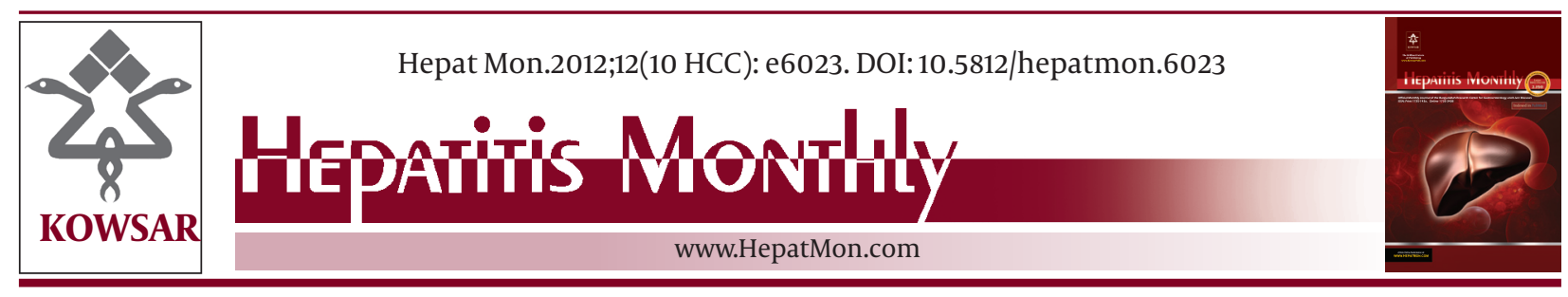

\title{
Hepatocellular Carcinoma in Pakistan: Where do We Stand?
}

\author{
Amna Subhan Butt ${ }^{*}$, Zaigham Abbas ${ }^{1}$, Wasim Jafri ${ }^{1}$ \\ ${ }^{1}$ Section of Gastroenterology, Department of Medicine, The Aga Khan University Hospital, Karachi, Pakistan
}

A R T I C L E I N F O

Article type:

Review Article

Article history:

Received: 29 Nov 2011

Revised: 16 Mar 2012

Accepted: 05 Apr 2012

\section{Keywords:}

Carcinoma, Hepatocellular

Pakistan

Hepatitis C

\begin{abstract}
A B S T R A C T
Context: From the 1970s till the mid 1990s, hepatitis B was the most common etiological factor for hepatocellular carcinoma (HCC) in Pakistan. Afterwards, a shift in HCC etiology was observed with a steady rise in hepatitis C virus (HCV) related HCC cases. HCV-3a, which is the most prevalent genotype, is also most frequent in HCV related HCC. There was an increase in the proportion of non-B non-C (NBNC) HCC cases as well, which might be attributed to an increase in non-alcoholic fatty liver disease.

Evidence Acquisition: The age-standardized rate for HCC is 7.64/100 000 in males and 2.8/100 000 in females. Male to female ratio is 3.6:1. Usual age of presentation is in the fifth and sixth decade. Most patients present with advanced disease, as they are not in a regular surveillance program. This is more so for patients with NBNC chronic liver disease. As many sonologists in Pakistan are practicing without sufficient training to pick up early lesions, alpha-fetoprotein is still recommended to compliment ultrasound in the surveillance of HCC.

Results: Majority of HCC patients present with nonresectable disease. Interventions such as transarterial chemoembolization, radiofrequency ablation, resection and chemotherapy including sorafenib are available in selected centers. Pakistan appears to be in an area of intermediate endemicity for HCC. There is a need for population based epidemiological studies to estimate the exact disease burden.

Conclusions: Measures to prevent the spread of hepatitis $C$ and $B$ can slow down the epidemic rise in the incidence of HCC in the coming decades. There is a need to implement a proper surveillance program to identify HCC cases at an early stage.
\end{abstract}

Published by Kowsar Corp, 2012. cc 3.0.

- Implication for health policy/practice/research/medical education:

This review article provides evidence gathered from published data related to the; magnitude, etiological factors, disease characteristics, therapeutic response and survival of HCC patients in Pakistan.

- Please cite this paper as:

Butt AS, Abbas Z, Jafri W. Hepatocellular Carcinoma in Pakistan; Where do We Stand?. Hepat Mon. 2012;12(10 HCC): e6023. DOI:10.5812/ hepatmon.6023

\section{Context}

Hepatocellular carcinoma (HCC) is the sixth most common cancer globally, attributing to 626000 or 5.7\% of new cancer cases annually (1). It is the third most common cause of cancer related deaths globally and carries an overall survival rate of only $3-5 \%$. The major burden of HCC lies in developing countries; up to $82 \%$ of HCC cases are reported from developing countries, including 55\% from China alone (1-3). Moreover, relatively high incidence rates have been found in South East Asia and in Sub-Saharan Africa (2-4). Hepatitis B and C are the major risk factors for HCC (5). Geographical variations in the significance of hepatitis B virus (HBV) and hepatitis C virus (HCV) infections in the development of HCC, clinical

\footnotetext{
* Corresponding author: Amna Subhan Butt, Department of Medicine, The Aga Khan University and Hospital Stadium Road, Karachi 74800, Pakistan. Tel: +92214930051, Fax:+92-214934294, E-mail: amna.subhan@aku.edu

DOI:10.5812/hepatmon.6023

Copyright@ 2012 Kowsar Corp. All right reserved.

This is an Open Access article distributed under the terms of the Creative Commons Attribution License (http://creativecommons.org/licenses/by/3.0), which permits unrestricted use, distribution, and reproduction in any medium, provided the original work is properly cited.
} 
features and survival have been reported $(1,6,7)$. However, the evidence regarding risk estimates for the development of HCC in individuals coinfected with HBV, HCV and hepatitis D (HDV) is lacking (3).

Pakistan is located in South-Asia where the prevalence of hepatitis B and C is intermediate $(6,7)$. Considering the burden of hepatitis $B$ and $C$, it is expected that the incidence of HCC will increase further in the foreseen future, especially in countries such as China and Taiwan (8). Hence, HCC will be a major burden on their healthcare systems (3). This review is based on evidence gathered from published data related to the; magnitude, etiological factors, disease characteristics, therapeutic response and survival of HCC patients in Pakistan.

\section{Evidence Acquisition}

\subsection{Search Strategy}

Medline indexed database, Google Scholar and a local search engine, i.e. PakMediNet (http://www.pakmedinet. com), which also lists local studies that are not listed in Medline) as well as abstracts from major international hepatology conferences, were used to search for relevant articles published during January 1970 till September 2011. Mesh terms; "Hepatocellular Carcinoma and Pakistan", "Hepatoma and Pakistan", "Primary liver cancer and Pakistan", "HCC and Pakistan" and "liver cancer and Pakistan" were used for the search. In addition, relevant studies were identified by reviewing the reference lists of selected articles. Those articles that were relevant and where their full text article or complete abstract was available and the sample size was at least 20 , were included in this review.

\section{Result}

\subsection{Epidemiology}

Unfortunately, no population based study was available from which a true prevalence and incidence rate of HCC could be ascertained. Most of the studies were hospital based, consisting of case series with small sample sizes or they had a highly select population. However, there have been a few cancer registries established in Pakistan. The Karachi Cancer Registry (KCR) was the first populationbased cancer registry, established in 1995, by the Sindh Government, in technical collaboration with the Unit of Descriptive Epidemiology, International Agency for Research on Cancer (IARC) of the World Health Organization (WHO) (9). The Aga Khan University Cancer Surveillance for Pakistan (ACSP) was established in 2000 at the Aga Khan University Pathology-based Cancer Registry (APCR), which covers a large geographical area and population of Pakistan, through their 84 centers. Moreover, APCR is an associate member of the IACR (10). The incidence and prevalence of the various cancers has been estimated in certain cities via these registries. Out of 4268 new cancer cases registered in the KCR from Karachi, dis- trict South during 1995-1997, the age-standardized rates (ASR) for HCC were found to be 5.7/100 000 in males and 3.7/100 000 in females (rank $9^{\text {th }}$ ) (9). Furthermore, ASR (world population was taken as a reference here) were found to be 5.3 and 4.0/100 000 in Karachi during 1998 to 2002 (11). Moreover, among the cancers registered to KCR and APCR from Larkana during 2000-2002, ASR for HCC were 10.5/100 000 persons in male and 2.0/100 000 in females (12). Likewise, cancer patients who were residents of Hyderabad and who were registered in KCR and APCR during 1998 to 2002, their ASR for HCC were reported to be 4.4/100 000 in males and 1.2/100 000 in females (10). From Quetta, 1077 cancers were registered in KCR during 1998 to 1999 . Here, HCC was found to be the third most common cancer in men (age standardized rate 12.3/100 000) and the seventh most common cancer in women (ASR 3.1/100 000) (13). Hence, ASR reported an average figure in these different studies as 7.64/100 000 for males and 2.8/100 000 for females. Another hospital based tumor registry has been established at the Armed Forces Institute of Pathology (AFIP). Out of 21168 cancers reviewed during 1992-2001, liver cancers were found in $22(2.81 \%)$ males and 22 (2.81\%) females (14).

Besides these registered data, 29 relevant studies were found where the epidemiological aspects of HCC were evaluated (Table 1). The numbers of cases from these 29 studies were aggregated $(n=3319)$ and trends of HCC, gender distribution and etiological factors were analyzed. Out of the 3319 cases, information regarding gender was available for 2967 cases. Out of 2967 cases, 78.29\% were male and $21.71 \%$ were female. Age ranges were between $8-98$ years. However, in most of the studies HCC cases presented during the fifth decade of life. Information about the etiological factors for HCC was available in 2852 cases; $\mathrm{HCV}, \mathrm{HBV}$ and $\mathrm{HCV} / \mathrm{HBV}$ co-infection was found in $57.99 \%$, $25.35 \%$ and $5.26 \%$ cases respectively. Coinfection with HBV and HDV was found in $1.09 \%$ cases and hepatitis B/C/D coinfection was found in $0.63 \%$ cases. Moreover, $9.68 \%$ cases were seronegative for hepatitis B and C (NBNC-HCC). As it is clear from Figure 1, the number of studies related to HCC increased with the passage of time. Hence, a rise in the number of HCC cases was observed during the study period. During the entire study, an almost consistent male preponderance was found in the HCC cases. As far as the etiology of HCC is concerned, it has been identified that from the 1970s till the mid 1990s, hepatitis B was the most common etiological factor for HCC. Afterwards, a shift in HCC etiology with a steady rise in HCV related HCC cases were observed (Figure 2). Moreover, an inconsistent rise in the number of HCC cases attributed to HBV/HCV or HCV/HBV/HDV co-infection and NBNC-HCC was also recognized (Figure 3). To evaluate the data further, we divided the time period into three categories; 1. 1970-1999 2. 2000-2005 and 3. 2006-2011, and the data was compared for these different time periods (Table 2). Again a clear, consistent shift was recognized in the etiology of 


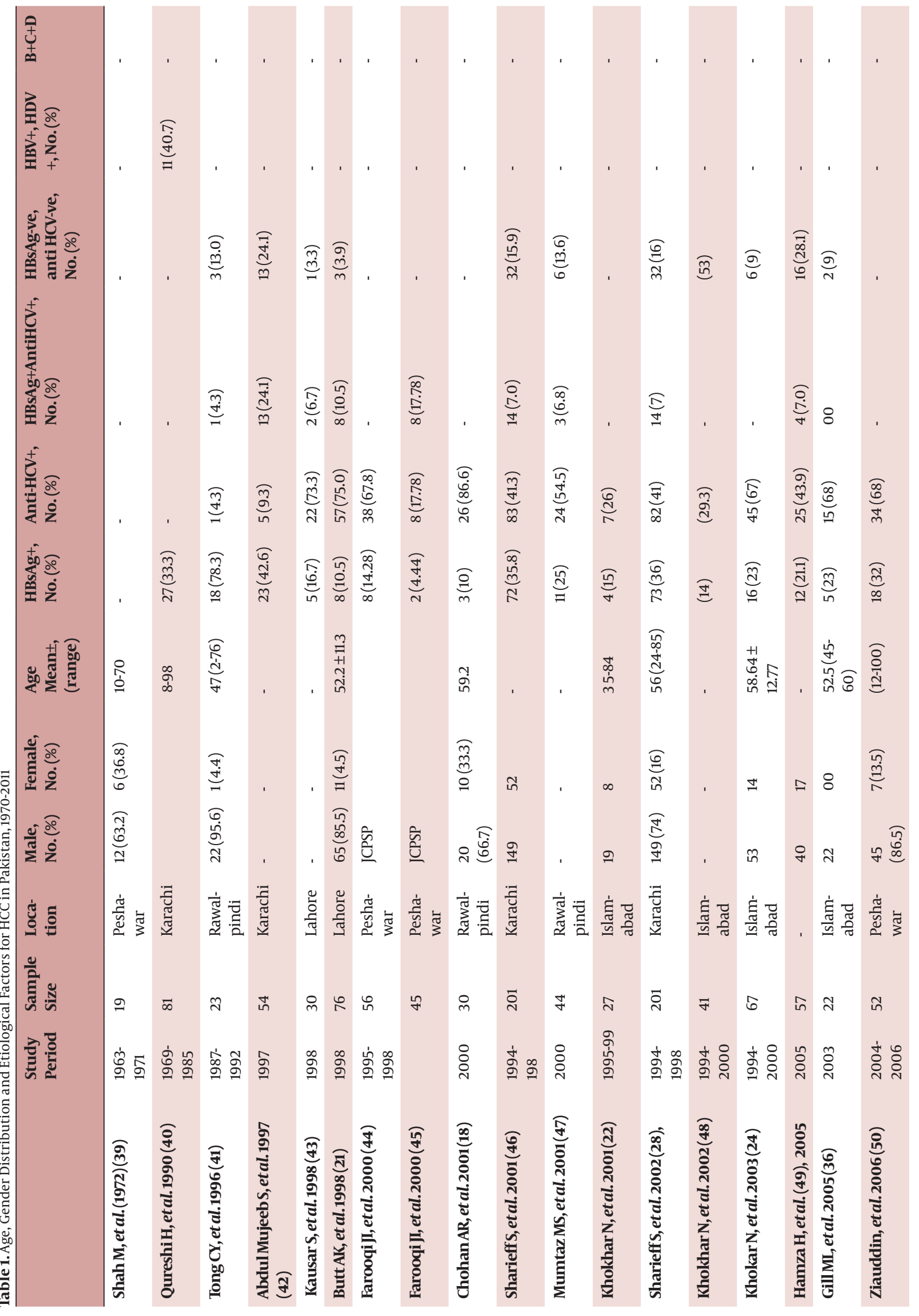




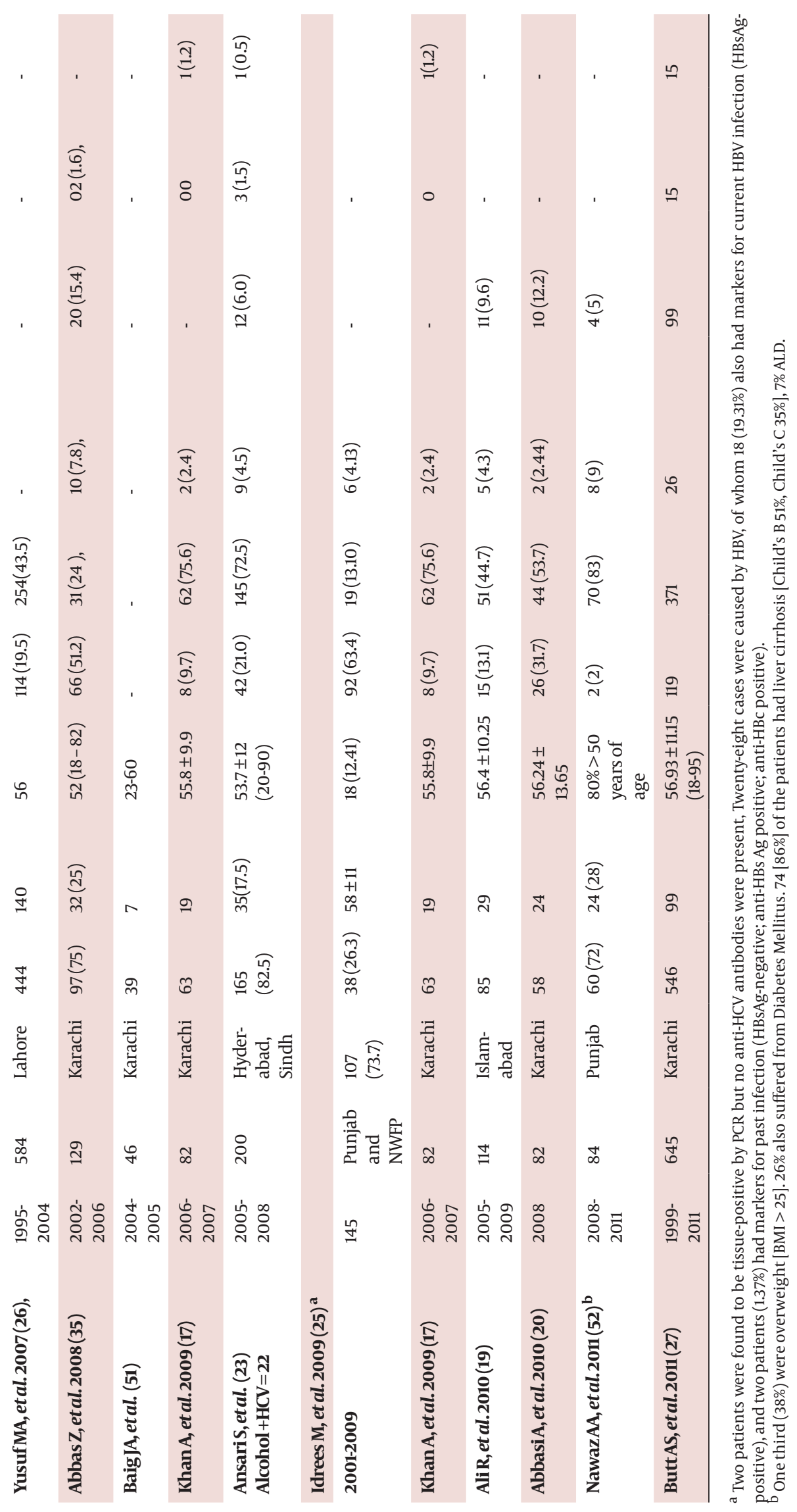


HCC (Figure 4). Replacement of HBV by HCV as the major etiological factor for HCC was quite clear. Moreover, a rising trend was seen in the number of NBNC-HCC cases during these time periods. Hence, viral hepatitis is identified as the major attributable factor of HCC in Pakistan. Hepatitis C followed by hepatitis B, HBV/HCV coinfection and HBV/HDV coinfection are the foremost factors leading to HCC. Considering the increasing prevalence of diabetes, hypertension and dyslipidemia in Pakistan, we can assume that the rising numbers of NBNC-HCC might be attributed to non-alcoholic fatty liver disease (NAFLD) which requires further studies for confirmation. Further-

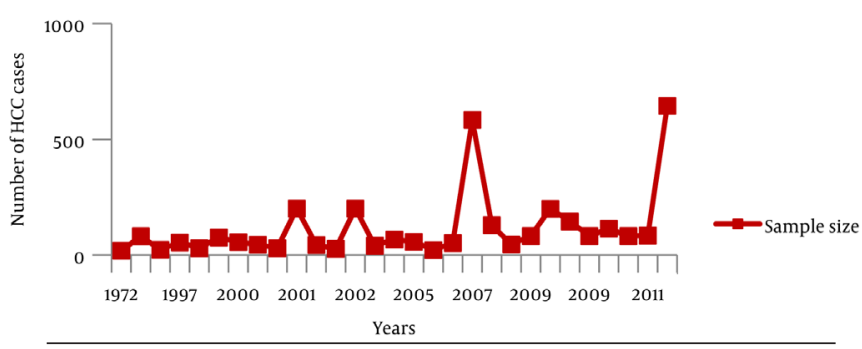

Figure 1. Distribution of HCC Cases, 1970-2011

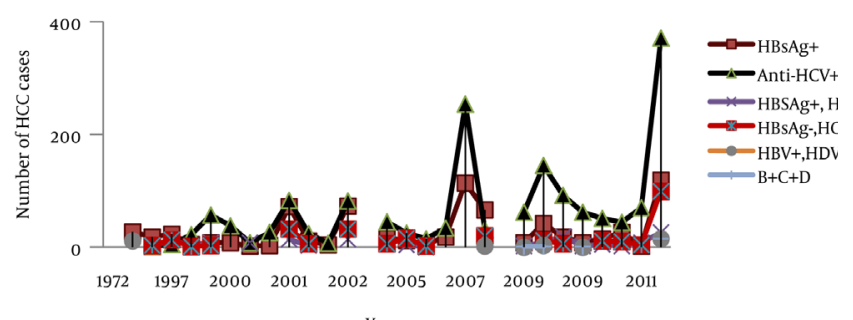

Years

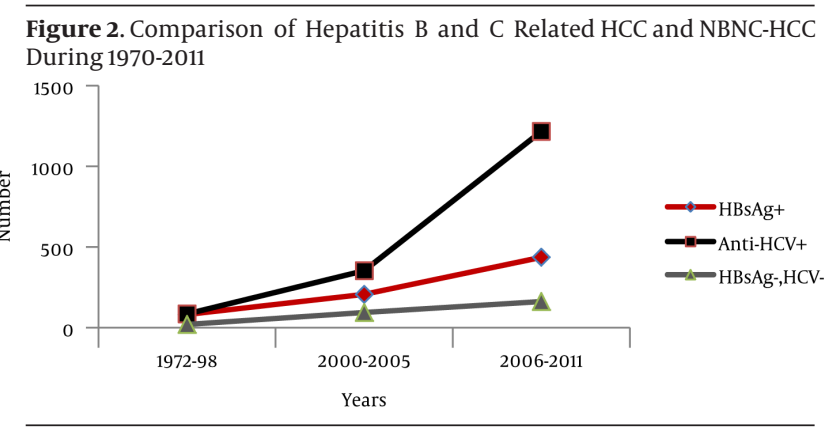

Figure 3. Comparison of Various Etiological Factors for HCC, 1970-2011

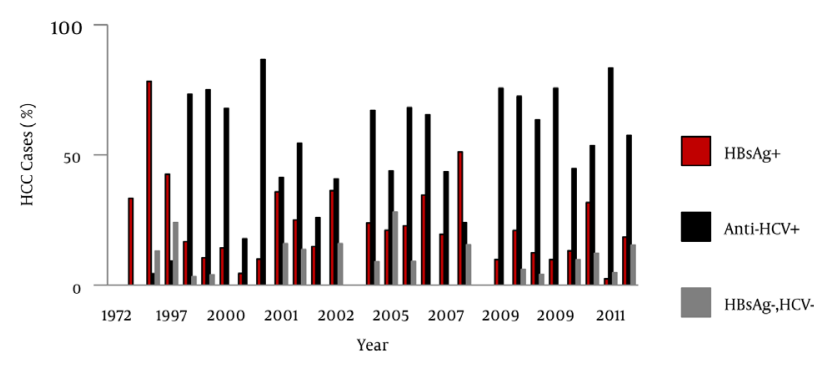

Figure 4. Comparison of Hepatitis B and C related HCC and NBNC-HCC during Different Time Intervals more, in Pakistan, a high rate of food contamination with aflatoxin has been reported earlier, and this is a known carcinogen for HCC in humans $(15,16)$. However, there is a lack of data regarding aflatoxins leading to HCC from Pakistan.

\subsection{HCV Genotypes and HCC}

Hepatitis $C$ is the commonest cause of HCC in most of the developing countries and the distribution of HCV genotypes varies across the world. Hence, studies have been conducted in different populations worldwide to evaluate the association of HCV genotype 1 with HCC development. In Pakistan, the most prevalent HCV genotype is 3. Until now, only two studies have been conducted in Pakistan to study the association of HCV genotypes with HCC. Khan A et al. (17) evaluated 189 patients with chronic liver disease including 82 with HCC. Hepatitis $C$ genotype 3a was the predominant genotype ( $81.4 \%)$ followed by $3 \mathrm{~b}$ (9.3\%), 3k (2.3\%), 1a (1.5\%), 1c (1.5\%), 1b (0.8\%), and 2a (0.8\%). Out of 82 HCC cases, $76 \%$ were infected with genotype $3 a$. However, considering the small sample size this study lacks the strength to correlate other HCV genotypes with HCC in our population. The additional information that was provided by the study from Khan et al.(17), is the existence of a distinct phylogenetic cluster of genotype 3a in Pakistan, and its appearance in this region in 1920s had a rapid exponential growth in the 1950s. Hence, these findings suggest an earlier epidemic spread of HCV-3a in Pakistan than in the other countries where genotype 3 has been reported. This might be associated with the increasing incidence of HCC in Pakistan during the last few decades. Likewise, in another study the distribution of the HCV genotype found was; $3 \mathrm{a}$ in $40.96 \%$, 3b in $15.66 \%$, 1a in $9.63 \%$, and $1 \mathrm{~b}$ in $2.40 \%$ and mixed genotypes in $28.91 \%$ of HCC patients. Of the 24 mixed genotypes, ten were infected with genotypes $3 \mathrm{a}$ and $3 \mathrm{~b}$, eight with $1 \mathrm{a}$ and $3 \mathrm{a}$, and six with $1 \mathrm{a}$ and $3 \mathrm{~b}$. While, two tissue samples were found to be untypable as no genotype was detected (37).

\subsection{Clinical Presentations of HCC}

Most of the available studies have emphasized the epidemiological aspects. In general most of the patients remained; asymptomatic or experienced right hypochondrial pain, weight loss, jaundice, fever, upper gastrointestinal bleed, hepatic encephalopathy, hepatosplenomegaly, abdominal/liver mass and ascites (18-23). Cirrhosis was reported in $69-84 \%$ cases with Child's class B or C in most of the cases $(18,21-25)$. Out of 400 HCC cases reviewed by Yusuf et al. (26) 216, 147 and 37 patients had Child's class A, B and C respectively. Whilst in another study, out of 645 HCC cases, the majority had Child's class B (42.3\%) or C (45.1\%) cirrhosis (27).

Furthermore, it was an alarming finding that $82.9 \%$ of the HCC cases in the largest case series of 645 patients were diagnosed when they were symptomatic and $8.8 \%$ 


\begin{tabular}{|c|c|c|c|c|c|c|c|c|c|}
\hline Year & Sample Size & Male & Female & HBsAg+ & Anti-HCV+ & HBSAg + \& HCV + & HBsAg- \& HCV- & $\mathrm{HBV}+\& \mathrm{HDV}+$ & B \& C \& D+ \\
\hline 1970-1999 & 283 & 99 & 18 & 81 & 85 & 24 & 20 & 11 & 0 \\
\hline 2000-2005 & 791 & 452 & 153 & 206 & 353 & 43 & 94 & 0 & 0 \\
\hline 2006-2011 & 2245 & 1772 & 473 & 436 & 1216 & 83 & 162 & 20 & 18 \\
\hline
\end{tabular}

\begin{tabular}{|c|c|c|c|c|c|}
\hline & Sample Size & Mean \pm SD or Median & Range & Others, ng/ml & Elevated AFP, \% \\
\hline Butt AK, et al. 1998 (21) & 76 & $142 \pm 155$ & $2.7-1470$ & - & - \\
\hline Shah GG, et al. 1999 (53) & 32 & - & - & - & 84.3 \\
\hline Khokhar N, 2001, et al. (22) & 27 & - & $43-6300$ & - & \\
\hline Chohan Arm, et al. 2001 (18) & 30 & - & - & - & 63.3 \\
\hline \multirow[t]{2}{*}{ Sharieff S, et al. 2001 (32) } & 201 & $17,027 \pm 68,853$ & - & $>1000: 35 \%$ & 35 \\
\hline & & & & Normal AFP: $24 \%$ & \\
\hline Khokar N, et al. 2003 (24) & 67 & $632.09 \pm 1332.31$ & - & - & 80 \\
\hline Gill ML, et al. 2005 (36) & 22 & $15000 \pm 1000$ & - & - & \\
\hline \multirow[t]{3}{*}{ Ziauddin, et al. 2006 (50) } & 52 & - & - & $<200: 13.5 \%$ & 100 \\
\hline & & & & $200-400: 25 \%$ & \\
\hline & & & & $>400 ; 61.5 \%$ & \\
\hline Yusuf MA, et al. 2007 (26) & 442 & $4198 \pm 262$ (median) & $1-278,560$ & $>200: 70 \%$ & - \\
\hline Abbas Z, et al. 2008 (35) & 129 & & & $>400: 37.5 \%$ & 31 \\
\hline Baig JA, et al. 2009 (51) & 39 & $421 \pm 59$ & $101-2341$ & - & - \\
\hline \multirow[t]{3}{*}{ Ali R, et al. 2010 (19) } & 114 & - & - & $<100: 7 \%$ & 72.8 \\
\hline & & & & $>100: 10.5 \%$ & \\
\hline & & & & $\geq 500: 5.3 \%$ & \\
\hline Abbasi AB, et al. 2010 (20) & 82 & 2582.52 & 2.54- 65609 & $\geq 400$ in $46.34 \%$ & - \\
\hline \multirow[t]{2}{*}{ Butt AS, et al. 2011 (27) } & 645 & 82.0 (median) & $0.95-303717$ & $\geq 20: 65.7 \%$ & 67.5 \\
\hline & & & & $\geq 200: 41.2 \%$ & \\
\hline
\end{tabular}

were diagnosed incidentally. Whereas, only $8.2 \%(n=53)$ of the HCC cases were diagnosed on screening. The duration between diagnosis of a chronic liver disease and HCC was $24.01 \pm 38.05$ months (range 0-195 months). Moreover, 480 (74.4\%) patients experienced at least one complication related to cirrhosis earlier to their index presentation, and that included; ascites (68.5\%), portosystemic encephalopathy (34.1\%), esophageal varices (53\%), upper gastrointestinal bleeding (40.5\%), hepatorenal syndrome (22.6\%), hepatohydrothorax (9\%), hepatopulmonary syndrome (7.8\%) and hypersplenism (62.2\%). Among all of the patients, 259 (40.2\%), $222(34.4 \%)$ and $16(2.5 \%)$ had concomitant diabetes, hypertension or dyslipidemia respectively (27). In some studies HBV-related HCC patients were found to be younger than HCV-related HCC patients (49.7 v/s 56.3 years) (28). Elevated serum bilirubin (100\%), alanine aminotransferase (ALT) (42.1\%), aspartate aminotransferase(AST) (42.1\%), and alkaline phosphatase (100\%), were reported in a group of 145 patients (25). Hence, most of the patients presented with an advanced disease stage, as they were not under a regular surveillance program. This was even true for patients with NBNC chronic liver disease leading to HCC (27). In the process of comparing HCC in HBV mono-infection with HBV/HDV co-infection, it was later found to be associated with a smaller liver size and indirect evidence of more severe portal hypertension in an earlier TNM stage (29).

\subsection{Diagnosis of HCC \\ 3.4.1. Alpha-Fetoprotein}

Alpha-fetoprotein ( $\alpha$-fetoprotein, AFP) is a large serum glycoprotein, used as a tumor marker for HCC. The summary of AFP in various studies is given in Table 3. A wide variability in ranges of AFP was found in the various studies. Elevated AFP was reported in $31-100 \%$ cases. However, $\mathrm{AFP}>200$ or $>400 \mathrm{ng} / \mathrm{ml}$ was found to be associated with a greater accuracy in detecting HCC. The diagnostic value of AFP for HCC, was evaluated among 100 biopsy proven HCC cases and 100 healthy subjects, who were found to 


\begin{tabular}{|c|c|c|c|c|}
\hline & Sample Size & Tumor Diameter Mean \pm SD, cm & No. of Lesion & Others \\
\hline \multirow[t]{2}{*}{ ButtAK, et al.1998(21) } & 76 & $7.8 \times 8.1$ & Solitary: $54 \%$ & $>8 \mathrm{~cm}: 54 \%$ \\
\hline & & & Multifocal/diffuse : $46 \%$ & \\
\hline \multirow[t]{2}{*}{ Shah GG, et al.1999(53) } & 32 & & Diffuse: $38 \%$ & $>10 \mathrm{~cm}: 31.2 \%$ \\
\hline & & & & $\begin{array}{l}\text { Right lobe involved } \\
\text { predominantly }\end{array}$ \\
\hline Sharieff S, et al.2001(32) & 201 & $8.3 \pm 4.0$ & - & $>5 \mathrm{~cm}: 79.49 \%$ \\
\hline \multirow[t]{2}{*}{ Khokhar N, et al.2001(22) } & 27 & & Single: $63 \%$ & - \\
\hline & & & Multiple: $37 \%$ & \\
\hline \multirow[t]{2}{*}{ KhokarN, et al.2003(24) } & 67 & $6.6 \pm 1.14$ & Single: $49 \%$ & $\begin{array}{l}\text { Rt /left lobe involved: } \\
3 / 7\end{array}$ \\
\hline & & & Multiple: 51\% & \\
\hline \multirow[t]{4}{*}{ Yaqoob J, et al.2004(34) } & 40 & 3.1 & Single: 13 cases & $\begin{array}{l}\text { Range for tumor size: } \\
0.8 \text { to } 14\end{array}$ \\
\hline & & & Multifocal: 13 cases & \\
\hline & & & $\begin{array}{l}\text { Dominant mass with } \\
\text { satellite lesions: } 12\end{array}$ & \\
\hline & & & $\begin{array}{l}\text { Cluster of contiguous } \\
\text { nodules: } 2\end{array}$ & \\
\hline Gill ML, et al. 2005 (36) & 22 & $5.0 \pm 1.0$ & & \\
\hline \multirow[t]{3}{*}{ Ziauddin, et al.2006(50) } & 52 & 5.41 & $>10 \mathrm{~cm} 15.4 \%$ & $\begin{array}{l}\text { Rt/left/both lobes in- } \\
\text { volved: } 44.2 \% / 5.8 \% / 50 \%\end{array}$ \\
\hline & & & $5.1-10 \mathrm{~cm}-28.8 \%$ & \\
\hline & & & $2-5 \mathrm{~cm}-55.8 \%$ & \\
\hline \multirow[t]{2}{*}{ Yusuf MA, et al.2007(26) } & 497 & 8 & Solitary: $33 \%$ & \\
\hline & & & Multifocal: $52 \%$ & \\
\hline \multirow[t]{3}{*}{ Ansari S, et al.2009(23) } & 200 & & Single $(<5 \mathrm{~cm}): 30 \%$ & PVT: $18 \%$ \\
\hline & & & Single $(>5 \mathrm{~cm}): 19.5 \%$ & \\
\hline & & & Multicentric: $68.34 \%$ & \\
\hline \multirow[t]{3}{*}{ Abbasi AB, et al.2010(20) } & 82 & & Single: $68.29 \%$ & PVT: $24.39 \%$ \\
\hline & & & Multiple: $26.82 \%$ & $\begin{array}{l}>50 \% \text { liver involved : } \\
51.21 \%\end{array}$ \\
\hline & & & Diffuse : $4.87 \%$ & \\
\hline \multirow[t]{6}{*}{ ButtAS, et al.2011(27) } & 645 & $5.62 \pm 3.67$ & Solitary: $38.1 \%$ & $<5 \mathrm{~cm}$ in $55.7 \%$ \\
\hline & & & Paucifocal: $40.2 \%$ & $5-10 \mathrm{~cm}$ in $33 \%$ \\
\hline & & & Multifocal:14.9\% & $>10 \mathrm{~cm}$ in $11.3 \%$ \\
\hline & & & Massive/infiltrative: $6.8 \%$ & PVT: $33.5 \%$ \\
\hline & & & & $\begin{array}{l}\text { Extrahepatic spread: } \\
13.2 \%\end{array}$ \\
\hline & & & & $\begin{array}{l}\text { Rt/left/both } \\
\text { lobes:60\%/12.3/27.5 }\end{array}$ \\
\hline
\end{tabular}


be HBsAg positive on blood screening (30). AFP was found with $72 \%$ sensitivity, $89 \%$ specificity, $86.7 \%$ positive predictive value, and $76.1 \%$ negative predictive value to detect HCC $(P<0.001)$. Likewise in another study $(n=100)$, AFP was found to be $72 \%$ sensitive and, $89 \%$ specific to detect HCC (31). However, no correlation was found between the size of the HCC and AFP levels when assessed in $201 \mathrm{pa}-$ tients $(r=-0.155 ; P=0.129)$ (32). There is much debate nowadays about the value of AFP in a surveillance program for HCC. What we have observed here in Pakistan is that many sonologists are practicing with insufficient training to pick up early lesions. Hence, alpha-fetoprotein is generally used as a screening tumor marker to compliment ultrasound liver tests for the surveillance of HCC.

\subsubsection{Radiological Features of HCC}

The presence of arterial enhancement, followed by washout of contrast in the portal-venous and delayed phase, are considered to be typical characteristic features of HCC $(19,33)$. Tumor size, number of lesions, local or distant metastasis are all prognostic factors and these help to make a decision about treatment. A summary of the various studies which describe radiological characteristics of HCC are given in Table 4. The role of biphasic contrast-enhanced helical CT including hepatic arterial phase (HAP) with portal venous phase (PVP) imaging, in the detection and characterization of HCC was evaluated by Yaqoob $\mathrm{J}$ et al. in 40 biopsy proven, HCC cases (34). The detection rate for HCC was $85 \%$ with HAP imaging (hyperattenuating $=69$, hypoattenuating $=3$ ) when compared to $48 \%$ with PVP imaging (hyperattenuating $=2$, hypoattenuating $=39)(P=0.008$. Moreover, in $7(17 \%)$ cases HCC was visible only in the HAP images.

\subsubsection{Liver Biopsy}

For the diagnosis of HCC, procedures such as; AFP, liver biopsy, triphasic CT scan and ultrasound of the abdomen have been evaluated in various studies. In a study by Yusuf et al. (26) these different diagnostic modalities were evaluated for HCC. Out of 584 patients, fine needle aspiration (FNA) of the liver lesion was done in 71 cases, a core biopsy in 26 and a lipoidal angiography was carried out in 42 patients. A combination of typical radiological findings on an imaging modality and an elevated AFP ( $>$ $200 \mathrm{ng} / \mathrm{ml}$ ) was found in 70 patients. Moreover, a combination of at least two of these modalities, i.e. characteristic findings on a triphasic CT scan, AFP level $>200 \mathrm{ng} / \mathrm{ml}$ and positive cytology or histology) was reported in 365 patients. FNA under ultrasound guidance was studied in 60 patients with suspected HCC. Mortality was reported in two cases secondary to uncontrollable bleeding after the procedure (21). In another study, out of 114 patients with suspected HCC, 48 (42.1\%) patients were found to have biopsy proven HCC. Histologically, well differentiated, moderately differentiated, poorly differentiated, lamellar HCC were reported in $26.4 \%, 34.5 \%, 30.9 \%$ and $8.2 \%$ patients respectively (27).

\subsection{Staging and Prognostic Factors for Survival}

Different staging systems have been used in different studies. In a study of 76 patients $22 \%, 61 \%$ and $17 \%$ were found to have Okuda stage I, II and III respectively (20). Abbas Z et al. (35) estimated the survival of 129 HCC patients. Median follow up was 11 months (range 2-36). Three patients were lost to follow-up. Cumulative deaths reported at six months, one year and at two years were; $22(21.6 \%), 62$ (60.8\%) and 93 (92.2\%) respectively. On univariate analysis; hepatitis $\mathrm{C}$ as etiology, female gender, presence of ascites, splenomegaly, splenic varices, INR > 1.3 , total bilirubin $>1.17 \mathrm{mg} / \mathrm{dl}$, direct bilirubin $>0.4 \mathrm{mg} /$ dl, alkaline phosphatase $>169 \mathrm{IU} / \mathrm{L}$, Model for End-Stage Liver Disease (MELD) Score $>12$, Child class B \& C, multifocal tumor, and transarterial chemoembolization (TACE) were not offered factors found to be associated with poor survival. However, on multivariate analysis, the overall independent determinants of poor survival were; hepatitis C as etiology, female sex and multifocality of tumor (hazard ratios 3.0, 3.0 and 1.9 respectively). Mean survival was 17.2 months for patients who underwent a TACE procedure, as compared to 12.8 months for those who did not receive TACE $(P=0.015)$. Okuda, Cancer of the Liver Italian Program (CLIP), Barcelona Clinic Liver Cancer (BCLC), Chinese University Prognostic Index (CUPI) and Child's staging systems retained their performance as judged by chi-square values in a regression analysis. Discriminatory ability for death, evaluated by the receiver operating characteristic curve, was better for the Okuda classification system in the first year.

\subsection{Treatment and Survival}

Unfortunately fewer studies were available regarding the treatment of HCC from Pakistan. In general, data regarding outcomes after curative therapies, i.e. liver transplantation, hepatic resection and radio frequency ablation, is not available. However, scanty data is available concerning the various palliative therapies. In a study by Yusuf et al. (12), only 79 out of 584 patients were found to be eligible for different modalities of treatment. Out of the 79, 48 patients underwent transarterial chemoembolization (TACE) using lipiodol and doxorubicin. Of these, 26 had disease progression, 11 had a stable disease state for a minimum of six months (range 6-20 months), and 11 patients were lost to follow-up. Local resection was done in 14 patients and 5 remained disease free for an average of 33 months. Percutaneous ethanol injection (PEI) was performed in 17 patients. Of these, 5 progressed within three months of treatment, 2 had a stable disease state for a mean of 13 months and 10 were lost to followup. Four patients underwent TACE followed by surgical resection. The overall median survival was 10.5 months. The cumulative probability of survival was $45 \%, 20 \%$ and $10 \%$ at 1-year, 3-years and 5-years. Median survival was bet- 
ter for patients with Child's class A (12 months) when compared to Child's B (7.7 months) and Child's C (4.1\%). Moreover, the difference in median survival between patients with Child's class A and B, or A and C was statistically significant $(P<0.001)(26)$. In the study by Abbas $Z$ et al. (35), 41 HCC patients underwent a TACE procedure. Overall the median survival rate was better for patients offered vs. not offered TACE (14.0 months vs. 9.5 months, OR 1.63, 95\% confidence interval 1.07-2.48, $P<0.05)$. TACE was found to be an effective palliative therapy in another group of 35 patients with unresectable HCC. The median survival time was 410 days. Moreover, a significant difference in mean survival time was found among the different Child's classes (p-value 0.002) (14). In another study of 201 patients, the median survival reported was only 16 weeks (28). Correlation between AFP level and survival was evaluated further. Survival was $17.6 \pm 11.5$ weeks for patients with an AFP level less than $10 \mathrm{ng} / \mathrm{ml}, 17.9 \pm 21.7$ weeks for patients with AFP levels between 10 to $1000 \mathrm{ng} / \mathrm{ml}$ and $13.2 \pm 15.6$ weeks for patients with an AFP level $>1000 \mathrm{ng} /$ $\mathrm{ml}$. However, this difference was not statistically significant. Moreover, no difference in survival rates was found for HBV or HCV related HCC (32).

Somatostatin receptors have been identified in HCC. Hence, long acting octreotide (LAR) has been tried in the treatment of HCC. In 2003, Gill et al. (36) evaluated the efficacy of LAR in the treatment of inoperable HCC in 22 patients, and the 20 patients who had refused the treatment were used as controls. Patients received 100 mcg octreotide (subcutaneously) thrice daily for two weeks, followed by monthly administration of $20 \mathrm{mg}$ intramuscular octreotide. A total of 19 patients completed the six month treatment. Regression in tumor size and reduction in mean AFP levels were reported in $45.5 \%$ and $50 \%$ of the cases respectively. Moreover, improvement in the quality of life at the end of treatment was seen in $45.5 \%$ cases. At the end of the six month treatment, $64 \%$ of the patients were alive in the intervention group as compared to $50 \%$ in the control group. In another study conducted by Frooqi et al. (37), 13 advanced HCC cases were randomized to receive either 250 ug subcutaneous octreotide twice daily (6 patients) or no treatment (7 patients). Significant improvement in the quality of life amongst the treatment group was observed, as compared to the control group $(P<0.05)$. In both studies, the authors recommended octreotide as an alternative for the treatment of inoperable HCC. However, both studies lacked the power to conclude that octreotide had a beneficial effect, due to the small sample sizes and possible selection bias. In a small series of 76 patients, 22 patients received intralesional alcohol injections. However, $54 \%$ of these patients with a tumor size $>8 \mathrm{~cm}$ died during follow up; this was due to liver failure in 8 cases and fatal bleeding occurred in 4 patients (21). In a study done by Anis et al., oral capecitabine monotherapy failed to halt tumor growth in advanced hepatocellular carcinoma (38). In general, studies regarding the curative treat- ment of HCC are not available. There are only a few cases that underwent resection of HCC which has been mentioned in several studies. Current existing data regarding treatment and prognosis carries the limitation of small sample size; it is mainly based upon single center experiences and lack of proper follow-ups. Hence, reporting the results of curative and various palliative treatment modalities will provide evidence regarding the outcome and natural history of our HCC cases.

\section{Conclusion}

Hepatitis $\mathrm{C}$ related chronic liver disease has become the major cause of a rising prevalence of HCC in Pakistan. The country appears to be in an area of intermediate endemicity for HCC. There is a need for population based epidemiological studies to estimate the exact disease burden. Measures to prevent the spread of hepatitis $C$ and $\mathrm{B}$ can slow down the epidemic rise in the incidence in the coming decades. There is a need to implement a proper surveillance program to pick up the disease early. Treatment facilities are not widely available as data about treatment outcomes is scarce. The availability of a sufficient number of patients opens opportunities to do translational research, however, this is lacking at the moment.

\section{Acknowledgments}

None declared.

\section{Authors' Contribution}

Butt AS conceived the study question, collected the data, performed the statistical analysis and wrote manuscript. Abbas $\mathrm{Z}$ and Jafri $\mathrm{W}$ participated in the design of the study and reviewed manuscript. All authors read and approved the final manuscript.

\section{Financial Disclosure}

None declared.

\section{Funding/Support}

None declared.

\section{References}

1. Parkin DM. The global health burden of infection-associated cancers in the year 2002. Int J Cancer. 2006;118(12):3030-44.

2. Parkin DM, Bray F, Ferlay J, Pisani P. Global cancer statistics, 2002. CA Cancer JClin. 2005;55(2):74-108.

3. Raza SA, Clifford GM, Franceschi S. Worldwide variation in the relative importance of hepatitis $B$ and hepatitis $C$ viruses in hepatocellular carcinoma: a systematic review. Br J Cancer. 2007;96(7):1127-34.

4. Ogunbiyi JO. Hepatocellular carcinoma in the developing world Semin Oncol. 2001;28(2):179-87.

5. Donato F, Boffetta P, MA P. Metaanalysis of epidemiological studies on the combined effect of Hepatitis B and C virus infections in causing hepatocellular carcinoma. Int J Cancer. 1998;75:347-54.

6. Andre F. Hepatitis B epidemiology in Asia, the Middle East and 
Africa. Vaccine. 2000;18 (Suppl 1):S20-2.

7. Jafri W, Subhan A. Hepatitis C in Pakistan: magnitude, genotype, disease characteristics and therapeutic response. Trop Gastroenterol. 2008;29(4):194-201.

8. Yuen MF, Hou JL, Chutaputti A. Hepatocellular carcinoma in the Asia pacific region.J Gastroenterol Hepatol. 2009;24(3):346-53.

9. Bhurgri Y, Bhurgri A, Hassan SH, Zaidi SH, Rahim A, Sankaranarayanan R, et al. Cancer incidence in Karachi, Pakistan: first results from Karachi Cancer Registry. Int J Cancer. 2000;85(3):325-9.

10. Bhurgri Y, Bhurgri A, Pervez S, Bhurgri M, Kayani N, Ahmed R, et al. Cancer profile of Hyderabad, Pakistan 1998-2002. Asian Pac J Cancer Prev. 2005;6(4):474-80.

11. Bhurgri Y. Karachi Cancer Registry Data-implications for the National Cancer Control Program of Pakistan. Asian Pac J Cancer Prev. 2004;5(1):77-82.

12. Bhurgri Y, Pervez S, Kayani N, Bhurgri A, Usman A, Bashir I, et al. Cancer profile of Larkana, Pakistan (2000-2002). Asian Pac J CancerPrev. 2006;7(4):518-21.

13. Bhurgri Y, Pervez S, Usman A, Khan JA, Bhurgri A, Kasi Q, et al. Cancer patterns in Quetta (1998-1999). J Pak Med Assoc 2002;52(12):560-5.

14. Jamal S, Moghal S, Mamoon N, Mushtaq S, Luqman M, Anwar $M$. The pattern of malignant tumours: tumour registry data analysis, AFIP, Rawalpindi, Pakistan (1992-2001). J Pak Med Assoc. 2006;56(8):359-62.

15. Nizami HM, Zuberi SJ. Aflatoxin and liver cancer in Karachi, a preliminary survey. J Pak Med Assoc. 1977;27(6):351-2.

16. Iqbal Q, Amjad M, Asi MR, Arino A. Assessment of hot peppers for aflatoxin and mold proliferation during storage. J Food Prot. 2011;74(5):830-5.

17. Khan A, Tanaka Y, Azam Z, Abbas Z, Kurbanov F, Saleem U, et al. Epidemic spread of hepatitis $C$ virus genotype $3 a$ and relation to high incidence of hepatocellular carcinoma in Pakistan. J Med Virol. 2009;81(7):1189-97.

18. Chohan AR UM, Khar B, Khurram M, Zahid M, Shah SF, et al. Demographic features of hepatocellular carcinoma: A study of 30 cases. J Rawalpindi Med Coll. 2001;5(2):81-3.

19. Ali R, Iqbal K, Irfan J. Characteristics of Hepatocellular Carcinoma Patients Attending NORI. Ann Pak Inst Med Sci. 2010;6(4):183-5

20. Abbasi A, Butt N, Bhutto AR, Gulzar K, SM M. Hepatocellular carcinoma: a clinicopathological study. J Coll Physicians Surg Pak. 2010;20(8):510-3.

21. Butt AK, Khan AA, Alam A, Ahmad S, Shah SW, Shafqat F, et al. Hepatocellular carcinoma: analysis of 76 cases. J Pak Med Assoc 1998;48(7):197-201.

22. Khokhar N. Multi locular presentation of hepatocellular carcinoma. JPMA. 2001;51(11):407.

23. Ansari S, Memon MS, Devrajani BR. Frequency of hepatitis B and hepatitis $C$ in patients with hepatocellular carcinoma at Hyderabad. JLUMHS. 2009;8(2):109.

24. Khokhar N, Aijazi I, Gill ML. Spectrum of hepatocellular carcinoma at Shifa International Hospital, Islamabad. J Ayub Med Coll Abbottabad. 2003;15(4):1-4.

25. Idrees M, Rafique S, Rehman I, Akbar H, Yousaf MZ, Butt S, et al. Hepatitis $\mathrm{C}$ virus genotype 3a infection and hepatocellular carcinoma: Pakistan experience. World J Gastroenterol. 2009;15(40):5080-5.

26. Yusuf MA, Badar F, Meerza F, Khokhar RA, Ali FA, Sarwar S, et al. Survival from hepatocellular carcinoma at a cancer hospital in Pakistan. Asian Pac J Cancer Prev. 2007;8(2):272-4

27. Butt AS, Hamid S, Ahmad A, Ghufran M, Javed AA, Ahmad B, et al. Hepatocellular carcinoma in Native South Asian Pakistani Population; Trends, Clinico-pathological characteristics \& differences in Viral Marker Negative \& Viral-HCC (A 10 year Experience). 9th Asian Pacific Association for the Study of the Liver (APASL) STC on HCC; 2011; Korea.

28. Sharieff S, Burney I, Salam A, Siddiqui T. Hepatocellular Carcinoma. J Coll Physicians Surg Pak. 2002;12(5):264-7.

29. Abbas Z, Qureshi M, Hamid S, Jafri W. Hepatocellular carcinoma in hepatitis D: Does it differ from hepatitis B monoinfection? Saudi J Gastroenterol. 2012;18(1):18-22.

30. Parvez T, Anwar MS. Diagnostic value of Alpha-Fetoprotein in liver cancer. J Coll Physicians Surg Pak. 2001;11(7):431-3.
31. Almani SA, Memon AS, Ghori RA, Memon J, Qureshi AF. AlphaFetoprotein as a diagnostic tool in differntiating Hepatocellular Carcinoma from benign hepatic disorders. J Liaquat Uni Med Health. 2004;3(1):7-12.

32. Sharieff S, Burney IA, Salam A, Siddiqui T. Lack of correlation between alpha-fetoprotein and tumor size in hepatocellular carcinoma. J Pak Med Assoc. 2001;51(3):123-4.

33. Bruix J, Sherman M. Management of hepatocellular carcinoma: an update. Hepatology. 2011;53(3):1020-2.

34. Yaqoob J, Bari V, Usman MU, Munir K, Mosharaf F, Akhtar W. The evaluation of hepatocellular carcinoma with biphasic contrast enhanced helical CT scan.J Pak Med Assoc. 2004;54(3):123-7.

35. Abbas Z, Siddiqui AU, Luck NH, Hassan M, Mirza R, Naqvi A, et al. Prognostic factors of survival in patients with non-resectable hepatocellular carcinoma: hepatitis $C$ versus miscellaneous etiology. J Pak Med Assoc. 2008;58(11):602-7.

36. Gill ML, Atiq M, Sattar S, Khokhar N. Treatment outcomes with long acting octreotide in inoperable hepatocellular carcinoma: a local experience and review of literature. J Pak Med Assoc. 2005;55(4):135-8.

37. Farooqi JI, Farooqi RJ. Efficacy of Octreotide in cases of inoperable hepatocellular carcinoma: A clinical trial. J Coll Phys Surg Pak. 2000;10:258-60.

38. Anis MK, Abbas Z, Jafri W. Oral Capecitabine for the treatment of advanced hepatocellular carcinoma. Pakistan J Gastroenterol. 2011.

39. Shah M, Mori W. Cirrhosis and primary hepatic cancer in Peshawar, Pakistan. A geographic pathological study. Bull Tokyo Med Dent Univ. 1972;19(2):165-77.

40. Qureshi H, Zuberi SJ, Jafarey NA, Zaidi SH. Hepatocellular carcinoma in Karachi. J Gastroenterol Hepatol.1990;5(1):1-6.

41. Tong CY, Khan R, Beeching NJ, Tariq WU, Hart CA, Ahmad N, et al. The occurrence of hepatitis $B$ and $C$ viruses in Pakistani patients with chronic liver disease and hepatocellular carcinoma. Epidemiol Infect. 1996;117(2):327-32.

42. Abdul Mujeeb S, Jamal Q, Khanani R, Iqbal N, Kaher S. Prevalence of hepatitis B surface antigen and HCV antibodies in hepatocellular carcinoma cases in Karachi, Pakistan. Trop Doct. 1997;27(1):45-6.

43. Kausar S, Shafqat F, Shafi F, Khan AA. The association of hepatocellular carcinoma with hepatitis B and C viruses. PakJ Gastroenterol.1998;12:1-2.

44. Farooqi JI, Farooqi RJ. Relative frequency of hepatitis B and C viruses infections in cases of hepatocellular carcinoma in North West Frontier Province, Pakistan. JColl Phy Surg Pak. 2000;10:12830 .

45. Farooqi JI, Farooqi RJ. Prevalence of Hepatocellular Carcinoma in Patients of Liver Cirrhosis: An Experience in North West Frontier Province (NWFP). J Coll Physicians Surg Pak. 2000;10(2):54-5.

46. Sharieff S, Burney KA, Ahmad N, Salam A, Siddiqui T. Radiological features of hepatocellular carcinoma in Southern Pakistan. Trop Doct. 2001;31(4):224-5.

47. Mumtaz MS, Iqbal R, Umar M, Khar B, Mumtaz MO, Anwar F, et al. Sero-prevalence of hepatitis B and C viruses in hepatocellular carcinoma. J Rawal Med Coll. 2001;5(2):78-80.

48. Khokhar N. Spectrum of chronic liver disease in a tertiary care hospital. J Pak Med Assoc. 2002;52(2):56-8.

49. Hamza N. Proc World Congress of Epidemiology 2005; Bangkok, Thailand.

50. Zia ud Din, Muhammad R, Saeedi MI, Mahmood K. Frequency of hepatoma in hepatitis B and C positive cirrhotic patients. J Postgrad Med Inst. 2006;20(4):325-29.

51. Baig JA, Alam JM, Mahmood SR, Baig M, Shaheen R, Sultana I, et al. Hepatocellular carcinoma (HCC) and diagnostic significance of A-fetoprotein (AFP). JAyub Med Coll Abbottabad. 2009;21(1):72-5.

52. Nawaz A, Chaudhry A, Riaz M, Yousaf M, Alvi A, Batool S, et al. Presentation of Hepatocellular Cancer in the Developing World: An Analysis of the Presentation and Association with Chronic Hepatitis B and C. AJG. 2011;106(supplement 2):S126.

53. Shah GG, Qureshi IA, Hakim T, Tarin BA, Farooq MA, Qureshi PS Radiological aspects of hepatocellular carcinoma: A hospital based study. Pak Armed Forces Med Jour. 1999;49:54. 